\title{
Conditions of Oscillation for a Linear Third-Order Differential Equation
}

\author{
Anees A. Fthee, Thair Y. Thanoon \\ Department of Mathematics, College of Computer and Mathematical Sciences University of Mosul, Al-Mosul, Iraq \\ Email: aneesaf79@gmail.com, thairyonis59@uomosul.edu.iq
}

How to cite this paper: Fthee, A.A. and Thanoon, T.Y. (2021) Conditions of Oscillation for a Linear Third-Order Differential Equation. Open Access Library Journal, 8: e7649.

https://doi.org/10.4236/oalib.1107649

Received: June 16, 2021

Accepted: August 20, 2021

Published: August 23, 2021

Copyright $\odot 2021$ by author(s) and Open Access Library Inc.

This work is licensed under the Creative Commons Attribution International License (CC BY 4.0).

http://creativecommons.org/licenses/by/4.0/

\section{(c) (i) Open Access}

\begin{abstract}
We introduce some new oscillation criteria for a third-order linear differential equation with variable coefficients in this study. We found out the corollary as a result of the Storm comparison theory and used it to prove some theorems. Through it, we were able to achieve the necessary conditions for oscillation. We concluded that the solution to the differential equation is oscillating if it $Q(x)$ is bounded from below, and also if the discriminant $D(x)$ of the equation is negative, its solution is oscillatory. We have given examples illustrating these results.
\end{abstract}

\section{Subject Areas}

Ordinary Differential Equation

\section{Keywords}

Oscillation Criteria, Oscillatory Solution

\section{Introduction}

The goal of this study is to develop oscillation conditions for a linear third-order differential equation with variable coefficients of the form:

$$
\frac{\mathrm{d}^{3} y(x)}{\mathrm{d} x^{3}}+m(x) \frac{\mathrm{d}^{2} y(x)}{\mathrm{d} x^{2}}+n(x) \frac{\mathrm{d} y(x)}{\mathrm{d} x}=0
$$

Throughout this work, we will solely focus on limitations on coefficient functions, namely $m(x)$ and $n(x)$, which are continuously differentiable functions on the interval $[\alpha, 1)$ and $\alpha$ is a non-negative real number. "The solution of the differential Equation (1) can be said to be non-trivial if at least in the infinite interval. The non-trivial solution of Equation (1) can be said to be oscillatory if it has arbitrarily large zeros on, otherwise, it is said to be non-oscillatory". We pre- 
sent some criteria for the oscillation behavior of third-order differential Equation (1). The work by ([1] [2] [3]) for the second order differential served as motivation for the results we give. We also give an example to show how the results presented in this research might be applied. Many criteria for the behavior of the integral of a combination of the coefficients of third order non-linear differential equations have been discovered. The authors were the ones who came up with this idea (for example see [4]-[12] and the authors therein).

\section{Main Results}

We shall deduce the Corollary from Sturm's Comparison Theorem and use it to establish certain theorems in this paper. First, we employ (Normal Form) to show that Equation (1) has an oscillation solution. We'll translate Equation (1) to Equation (5) as a flowing:

Let:

$$
\begin{gathered}
\frac{\mathrm{d} y(x)}{\mathrm{d} x}=\mathrm{e}^{-\frac{1}{2} \int m(x) \mathrm{d} x}\left[\frac{\mathrm{d} u(x)}{\mathrm{d} x}\right] \\
\frac{\mathrm{d}^{2} y(x)}{\mathrm{d} x^{2}}=\mathrm{e}^{-\frac{1}{2} \int m(x) \mathrm{d} x}\left[\frac{\mathrm{d}^{2} u(x)}{\mathrm{d} x^{2}}-\frac{1}{2} m(x) \frac{\mathrm{d} u(x)}{\mathrm{d} x}\right] \\
\frac{\mathrm{d}^{3} y(x)}{\mathrm{d} x^{3}}=\mathrm{e}^{-\frac{1}{2} \int m(x) \mathrm{d} x}\left[\frac{\mathrm{d}^{3} u(x)}{\mathrm{d} x^{3}}-m(x) \frac{\mathrm{d}^{2} u}{\mathrm{~d} x^{2}}-\frac{1}{2} m^{\prime}(x) \frac{\mathrm{d} u(x)}{\mathrm{d} x}+\frac{1}{4} m^{2}(x) \frac{\mathrm{d} u(x)}{\mathrm{d} x}\right]
\end{gathered}
$$

Substituting Equations (2), (3) and (4) into Equation (1) we get:

$$
\begin{gathered}
\mathrm{e}^{-\frac{1}{2} \int m(x) \mathrm{d} x}\left[\frac{\mathrm{d}^{3} u}{\mathrm{~d} x^{3}}-m(x) \frac{\mathrm{d}^{2} u}{\mathrm{~d} x^{2}}-\frac{1}{2} m^{\prime}(x) \frac{\mathrm{d} u}{\mathrm{~d} x}+\frac{1}{4} m^{2}(x) \frac{\mathrm{d} u}{\mathrm{~d} x}\right. \\
\left.+m(x)\left(\frac{\mathrm{d}^{2} u}{\mathrm{~d} x^{2}}-\frac{1}{2} m(x) \frac{\mathrm{d} u}{\mathrm{~d} x}\right)+n(x) \frac{\mathrm{d} u}{\mathrm{~d} x}\right]=0 \\
\mathrm{e}^{-\frac{1}{2} \int m(x) \mathrm{d} x}\left[\frac{\mathrm{d}^{3} u}{\mathrm{~d} x^{3}}--\frac{1}{2} m^{\prime}(x) \frac{\mathrm{d} u}{\mathrm{~d} x}-\frac{1}{4} m^{2}(x) \frac{\mathrm{d} u}{\mathrm{~d} x}+n(x) \frac{\mathrm{d} u}{\mathrm{~d} x}\right]=0 \\
\frac{\mathrm{d}^{3} u(x)}{\mathrm{d} x^{3}}-\frac{1}{2} m^{\prime}(x) \frac{\mathrm{d} u(x)}{\mathrm{d} x}-\frac{1}{4} m^{2}(x) \frac{\mathrm{d} u(x)}{\mathrm{d} x}+n(x) \frac{\mathrm{d} u(x)}{\mathrm{d} x}=0 \\
\frac{\mathrm{d}^{3} u(x)}{\mathrm{d} x^{3}}-\frac{1}{4}\left[m^{2}(x)-4 n(x)+2 m^{\prime}(x)\right] \frac{\mathrm{d} u(x)}{\mathrm{d} x}=0 \\
\frac{\mathrm{d}^{3} u(x)}{\mathrm{d} x^{3}}+Q(x) \frac{\mathrm{d} u(x)}{\mathrm{d} x}=0, Q(x)=-\frac{1}{4}\left[m^{2}(x)-4 n(x)+2 m^{\prime}(x)\right]=0
\end{gathered}
$$

where $Q(x)$ is continuous real value on $[\alpha, \infty), \alpha>0$.

Now we will give the main corollary, which we will rely on to fulfill the conditions of oscillation in the theorems of this paper.

\subsection{Corollary}

Let $y_{1}(x), y_{2}(x)$ are a nontrivial solutions on interval $I$ of the following dif- 
ferential equations:

$$
\frac{\mathrm{d}^{3} y(x)}{\mathrm{d} x^{3}}+Q_{1}(x) \frac{\mathrm{d} y(x)}{\mathrm{d} x}=0, \frac{\mathrm{d}^{3} y(x)}{\mathrm{d} x^{3}}+Q_{2}(x) \frac{\mathrm{d} y(x)}{\mathrm{d} x}=0
$$

Sequential, such that:

1) $Q_{1}(x)>Q_{2}(x), \forall x \in I$.

2) $y_{2}(x) y_{1}^{\prime}(x)>y_{1}(x) y_{2}^{\prime}(x), \forall x \in I$.

3) $W\left(y_{1}^{\prime}(x), y_{2}^{\prime}(x)\right) \geq 0, \forall x \in I$.

Then there are at last one zero of $y_{1}(x)$ between every two zeros of $y_{2}(x)$.

Proof:

Let $a, b$ are two zeros of $y_{2}(x)$ in $I$ such that $a<b$, suppose that $y_{1}(x) \neq 0$ for all $x \in(a, b)$. Since the zeros of $y$ is the same zeros of $-y$, therefore we can assume that every $y_{1}, y_{2}$ are positive in $(a, b)$. Since $y_{1}, y_{2}$ are linear independent on $I$ and $y_{2}(a)=y_{2}(b)=0$, therefore weget :

$$
w(b)=y_{1}(b) \cdot y_{2}^{\prime}(b) \text { and } w(a)=y_{1}(a) \cdot y_{2}^{\prime}(a)
$$

But

$$
\begin{gathered}
\frac{\mathrm{d}}{\mathrm{d} x} w\left(y_{1}(x), y_{2}(x)\right)=y_{1}(x) \cdot y_{2}^{\prime \prime}(x)-y_{2}(x) \cdot y_{1}^{\prime \prime}(x) \\
\frac{\mathrm{d}^{2}}{\mathrm{~d} x^{2}} w\left(y_{1}(x), y_{2}(x)\right) \\
=y_{1}(x) \cdot y_{2}^{\prime \prime \prime}(x)+y_{2}^{\prime \prime}(x) y_{1}^{\prime}(x)-y_{2}(x) \cdot y_{1}^{\prime \prime \prime}(x)-y_{1}^{\prime \prime}(x) y_{2}^{\prime}(x) \\
=-y_{1}(x) \cdot Q_{2}(x) y_{2}^{\prime}(x)+y_{2}^{\prime \prime}(x) y_{1}^{\prime}(x)+y_{2}(x) \cdot Q_{1}(x) y_{1}^{\prime}(x)-y_{1}^{\prime \prime}(x) y_{2}^{\prime}(x) \\
=y_{1}^{\prime}(x) y_{2}^{\prime \prime}(x)-y_{2}^{\prime}(x) y_{1}^{\prime \prime}(x)+y_{2}(x) \cdot Q_{1}(x) y_{1}^{\prime}(x)-y_{1}(x) Q_{2}(x) y_{2}^{\prime}(x) \\
=W\left(y_{1}^{\prime}(x), y_{2}^{\prime}(x)\right)+y_{2}(x) Q_{1}(x) y_{1}^{\prime}(x)-y_{1}(x) Q_{2}(x) y_{2}^{\prime}(x) \\
\frac{\mathrm{d}^{2}}{\mathrm{~d} x^{2}} w\left(y_{1}^{\prime}(x), y_{2}^{\prime}(x)\right)>0, \text { since, } Q_{1}(x)>Q_{2}(x)
\end{gathered}
$$

Therefore $W$ is increase on $(a, b)$. Since $y_{2}$ is positive function in this interval and its zeros $a, b$, hence,

$$
y_{2}^{\prime}(a) \geq 0 \text { and } y_{2}^{\prime}(b) \leq 0
$$

From Equation (6) we get:

$$
w(a) \geq 0 \text { and } w(b) \leq 0
$$

This will give us contradiction because $w$ is increase in interval $(a, b)$. Therefore must be have at lastone zero between $(a, b)$.

In the following theorem, we will understand the necessary condition for the oscillation of Equation (1), which will represent the first measure in this paper.

\subsection{Theorem}

If $Q(x)$ is a bounded below by a positive number on infinite interval $[\alpha, \infty)$. Then the a nontrivial solution of Equation (5) is oscillation on $[\alpha, \infty)$.

\section{Proof:}

Let $y(x)$ is a nontrivial solution of Equation (5). Since $Q(x)$ is a bounded 
below by a positive number on interval $I$. Then $\exists \varepsilon>0$ such that:

$$
Q(x)>\varepsilon^{2}>0, \forall x \in I
$$

Now, by use normal form of oscillation of Equation (5) we get:

$$
\frac{\mathrm{d}^{3} u(x)}{\mathrm{d} x^{3}}-\frac{1}{4}\left[m^{2}(x)-4 n(x)+2 m^{\prime}(x)\right] \frac{\mathrm{d} u(x)}{\mathrm{d} x}=0
$$

Compare Equation (7) with the flowing equation:

$$
\frac{\mathrm{d}^{3} u(x)}{\mathrm{d} x^{3}}+\varepsilon^{2} \frac{\mathrm{d} u(x)}{\mathrm{d} x}=0
$$

By corollary (2.1) the nontrivial solution $y_{1}(x)$ of Equation (7) fade at least once between two consecutivezeros of solution $y_{2}(x)$ Equation (8). Since $I$ is infinite interval therefore $y_{2}(x)$ has infinite number of zeros. So $y_{1}(x)$ will fade infinite times in $[\alpha, \infty)$. Then $y_{1}(x)$ will be oscillation on $[\alpha, \infty)$.

In the following example, we will show the fulfillment of the necessary condition for the fluctuation of the solution of Equation (1) after converting it to the form of Equation (5).

\subsection{Example}

Discuss the oscillation of the 3rd ODE, which has variable coefficients and is expressed as:

$$
\frac{\mathrm{d}^{3} y(x)}{\mathrm{d} x^{3}}-\frac{1}{x} \frac{\mathrm{d}^{2} y(x)}{\mathrm{d} x^{2}}+\frac{15}{x} \frac{\mathrm{d} y(x)}{\mathrm{d} x}=0
$$

\section{Solution:}

$$
\begin{gathered}
Q(x)=-\frac{1}{4}\left[m^{2}(x)-4 n(x)+2 m^{\prime}(x)\right] \\
Q(x)=-\frac{1}{4}\left[\frac{1}{x^{2}}-4\left(\frac{14}{x}\right)+\frac{2}{x^{2}}\right] \Rightarrow Q(x)=-\frac{1}{4}\left[-\left(\frac{60}{x}\right)+\frac{3}{x^{2}}\right] \\
Q(x)=\left[\frac{60}{4 x^{2}}-\frac{3}{4 x^{2}}\right] \Rightarrow Q(x)=\left[\frac{57}{4 x^{2}}\right] \\
\frac{\mathrm{d}^{3} u(x)}{\mathrm{d} x^{3}}+\left(\frac{57}{4 x^{2}}\right) \frac{\mathrm{d} u(x)}{\mathrm{d} x}=0, \text { Then } \exists \varepsilon>0 \text { such that: } Q(x)>\varepsilon^{2}>0, \forall x \in I \\
y(x)=1+2 x^{\frac{3}{2}} \operatorname{BesselJ}(3,2 \sqrt{15} \sqrt{x})+2 x^{\frac{3}{2}} \operatorname{BesselY}(3,2 \sqrt{15} \sqrt{x})
\end{gathered}
$$

In the following theorem, the proof will depend on the discriminant function of the Equation (1), which is $D(x)=\left|m^{2}(x)-4 n(x)+2 m^{\prime}(x)\right|$.

\subsection{Theorem}

If $D(x)<\alpha$ where $\alpha<0$ on $[\alpha, \infty)$. Then a nontrivial solution of Equation (5) is oscillation on $[\alpha, \infty)$. 


\section{Proof:}

Let $D(x)<\alpha$ where $\alpha<0$. This lead that $D(x)<0$. Then we get $Q(x)>0$. Therefore $Q(x)$ is a bounded below and by theorem (2.2) will be a nontrivial solution $y(x)$ of Equation (5) is oscillation on $[\alpha, \infty)$.

\subsection{Example}

Discuss the oscillation of the following $3^{\text {nd }} \mathrm{ODE}$, with variable coefficients, given as:

$$
\frac{\mathrm{d}^{3} y(x)}{\mathrm{d} x^{3}}-\frac{3}{x} \frac{\mathrm{d}^{2} y(x)}{\mathrm{d} x^{2}}+\frac{1}{8 x} \frac{\mathrm{d} y(x)}{\mathrm{d} x}=0
$$

\section{Solution:}

$$
\begin{gathered}
D(x)=\left|m^{2}(x)-4 n(x)+2 m^{\prime}(x)\right| \\
D(x)=\left[\frac{9}{x^{2}}-4\left(\frac{1}{8 x}\right)+2\left(\frac{3}{x^{2}}\right)\right] \Rightarrow D(x)=\left[\frac{15}{x^{2}}-\frac{1}{2 x}\right] \\
D(x)=\left[\frac{30}{2 x^{2}}-\frac{x}{2 x^{2}}\right] \Rightarrow D(x)=\left[\frac{30-x}{2 x^{2}}\right]
\end{gathered}
$$

Then $D(x)<0, \forall x>30$

Therefore the a nontrivial solution $y(x)$ is oscillation on $[\alpha, \infty)-\{x: x<0\}$.

$$
y(x)=1+2 x^{\frac{5}{2}} \operatorname{BesselJ}\left(5, \frac{1}{2} \sqrt{2} \sqrt{x}\right)+2 x^{\frac{5}{2}} \operatorname{Bessel}\left(5, \frac{1}{2} \sqrt{2} \sqrt{x}\right)
$$

\subsection{Theorem}

If $D(x) \geq 0$ for Equation (5) on $[\alpha, \infty)$. Then a nontrivial solution of Equation (5) $y(x)$ is non-oscillation on $[\alpha, \infty)$.

Proof:

If $D(x)=0$ then $Q(x)=0$ in the Equation (5) we will get:

$$
\frac{\mathrm{d}^{3} u(x)}{\mathrm{d} x^{3}}=0
$$

So the solation will be $y_{1}(x)=A x^{2}+B x+c$ where $A, B$ and $C$ are constant. This lead that the a nontrivial solution of Equation (9) will be symmetric of $y$-axes and have one zero at most. Now, if $D(x)>0$ on $[\alpha, \infty)$. then $Q(x)<0$ in the equation on $[\alpha, \infty)$.

$$
\frac{\mathrm{d}^{3} u(x)}{\mathrm{d} x^{3}}-Q(x) \frac{\mathrm{d} u(x)}{\mathrm{d} x}=0
$$

By compare Equation (9) with Equation (10). If a solution of Equation (10) $y_{2}(x)$ is oscillation on $[\alpha, \infty)$ by corollary (2.1) the nontrivial solution $y_{1}(x)=A x^{2}+B x+c$ has zero between two consecutive zeros of solution $y_{2}(x)$ and this is impossible because is symmetric of $y$-axes. There for any solution of Equation (1) is not oscillation on $[\alpha, \infty)$. 


\section{Conclusion}

We discovered the Corollary from Sturm's Comparison Theorem and used it to establish certain theorems in this work. We also explored the oscillatory solutions for third-order linear ordinary differential equations and estimated sufficient conditions of oscillation by converting Equation (1) to Equation (5). In general, we will extend this work to fourth-order linear ordinary differential equations in the future.

\section{Acknowledgement}

I would like to extend my thanks to the University of Mosul, College of Computer Science and Mathematics, Department of Mathematics.

\section{Conflicts of Interest}

The authors declare no conflicts of interest.

\section{References}

[1] Abdullah, H.K. (2004) A Note on the Oscillation of Second Order Differential Equations. Czechoslovak Mathematical Journal, 54, 949-954. https://doi.org/10.1007/s10587-004-6443-3

[2] Brtušek, M. (1999) On Oscillatory Solutions of Third Order Differential Equation with Quasiderivatives. Electronic Journal of Differential Equations, 3, 1-11.

[3] Kim, R.J. (2011) Oscillation Criteria of Differential Equations of Second Order. Korean Journal of Mathematics, 19, 309-319. https://doi.org/10.11568/kjm.2011.19.3.309

[4] Aktaş, M.F., Çakmak, D. and Tiryaki, A. (2011) On the Qualitative Behaviors of Solutions of Third Order Nonlinear Differential Equations. Computers \& Mathematics with Applications, 62, 2029-2036. https://doi.org/10.1016/j.camwa.2011.06.045

[5] Aktas, M.F., Tiryaki, A. and Zafer, A. (2009) Integral Criteria for Oscillation of Third Order Nonlinear Differential Equations. Nonlinear Analysis. Theory, Methods \& Applications, 71, e1496-e1502. https://doi.org/10.1016/j.na.2009.01.194

[6] Akmak, D.C. (2008) Oscillation for Second Order Nonlinear Differential Equations with Damping. Dynamic Systems and Applications, 17, 139.

[7] Cecchi, M., Došlá, Z. and Marini, M. (1997) Some Properties of Third Order Differential Operators. Czechoslovak Mathematical Journal, 47, 729-748. https://doi.org/10.1023/A:1022878804065

[8] Parhi, N. and Padhi, S. (2000) Asymptotic Behaviour of a Class of Third Order Delay-Differential Equations. Mathematica Slovaca, 50, 315-333.

[9] Parhi, N. and Das, P. (1992) Oscillation Criteria for a Class of Nonlinear Differential Equations of Third Order. Annales Polonici Mathematici, 57, 219-229. https://doi.org/10.4064/ap-57-3-219-229

[10] Parhi, N. and Das, P. (1998) Oscillatory and Asymptotic Behaviour of a Class of Nonlinear Differential Equations of Third Order. Acta Mathematica Scientia, 18, 95-106. https://doi.org/10.1016/S0252-9602(17)30694-X

[11] Parhi, N. and Das, P. (1998) On the Oscillation of a Class of Linear Homogeneous 
Third Order Differential Equations. Archivum Mathematicum, 34, 435-443.

[12] Škerlík, A. (1995) Integral Criteria of Oscillation for a Third Order Linear Differential Equation. Mathematica Slovaca, 45, 403-412. 